\title{
SAÚDE MENTAL E INTERVENÇÕES PSICOLÓGICAS DURANTE A PANDEMIA DA COVID-19: UM PANORAMA
}

\section{MENTAL HEALTH AND PSYCHOLOGICAL INTERVENTIONS DURING THE COVID-19 PANDEMIC: A PANORAMA}

\author{
ELIZEU BORLOTI ${ }^{1}$ - ORCID 0000-0002-6217-6541 \\ VERÔNICA BENDER HAYDU² - ORCID 0000-0002-4522-8338 \\ NÁDIA KIENEN ${ }^{2}$ - ORCID 0000-0003-2179-3700 \\ MARCELA ROBERTA JACYNTHO ZACARIN² - ORCID 0000-0001-5799-9458 \\ 1 UNIVERSIDADE FEDERAL DO ESPÍRITO SANTO \\ ${ }^{2}$ UNIVERSIDADE ESTADUAL DE LONDRINA
}

Impactos da COVID-19 sobre a saúde mental foram considerados no presente estudo, com o objetivo de avaliar aspectos relativos ao controle da disseminação da doença, à adesão aos procedimentos de tratamento e de prevenção, e às intervenções psicológicas de escolha. Foi realizada uma revisão narrativa da literatura, tendo sido abordadas descrições do impacto de epidemias no comportamento e na saúde mental da população em geral e dos profissionais da saúde. Além disso, foram descritas as intervenções psicológicas de escolha aos principais problemas de saúde mental em pandemias, incluindo aspectos gerais de sua aplicação remota. Os sintomas e transtornos mais frequentemente observados e que requerem intervenções são estresse agudo, estresse pós-traumático e desregulação emocional (envolvendo ansiedade, raiva e depressão). É relevante enfatizar contingências envolvendo a incontrolabilidade dos fatos e a gravidade dos eventos como precipitantes ou potencializadores desses sintomas e transtornos. Portanto, elas devem ser consideradas por aqueles que usam a mídia para promover mudanças no comportamento da população em relação a doenças infecciosas pandêmicas. Esses resultados revelam a importância da prevenção da contaminação, do tratamento médico das pessoas infectadas e do apoio psicológico às famílias e aos profissionais de saúde.

Palavras-chave: COVID-19; saúde mental; adesão; tratamento psicológico.

Impacts of the COVID-19 on mental health were considered in the present study, to evaluate aspects concerning the control of the spread of the disease, adherence to treatment and prevention procedures, and psychological interventions of choice. A narrative review of the literature was carried out, to describe the impact of epidemics on behavior and mental health of the general population and health professionals. It also describes the psychological interventions of choice for relevant mental health problems in pandemics, including general aspects of their remote application. The most frequently observed symptoms and disorders requiring interventions are acute stress, post-traumatic stress, and emotional dysregulation (involving anxiety, anger, and depression). Contingencies involving the uncontrollability of facts and the seriousness of events must be emphasized due to their accountability in precipitating or potentiating these symptoms and disorders. Therefore, they should be considered by those who use the media to promote changes in the behavior of the population concerning pandemic infectious diseases. These results highlight the relevance of contamination prevention, medical treatment of infected people, and psychological support to families and health professionals.

Keywords: COVID-19; mental health; adherence; psychological treatment.

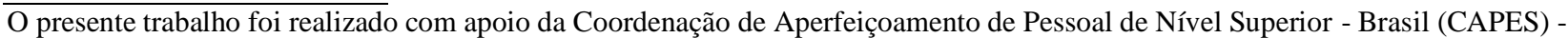
Código de Financiamento 001. Correspondência relativa a este manuscrito deve ser endereçada a veronicahaydu@gmail.com DOI: http://dx.doi.org/10.18542/rebac.v16i1.8885 
As doenças endêmicas e epidêmicas impactam níveis diferentes da vida humana; sua evolução como pandemia configura esses impactos como crise mundial. No nível macrossocial, por exemplo, políticas e economias são afetadas; no microssocial, famílias, escolas e vizinhança mudam suas práticas; no nível individual, exige-se mais auto-observação de sintomas e autocuidado com a saúde. A extensão da crise de uma pandemia em seus efeitos na vida de todas as pessoas, desde a infância até a terceira idade, precisa ser analisada e seus problemas solucionados de modo a evitar, conforme apontaram Levin, Gebbie e Qureshi (2007), uma crise social ou o abalo de estruturas, como o colapso do sistema de saúde de uma sociedade.

Neste ano de 2020, a extensão dessa crise é consideravelmente ampla. Seu impacto está sendo presenciado em vários países, conforme foi constatado a partir do anúncio, em 11 de março de 2020 pela Organização Mundial da Saúde, da pandemia da COVID19 - abreviação de COrona VIrus Disease, surgida em 2019. Antes desse anúncio, a pandemia da COVID-19 já havia atingido 114 países. Antes ainda, outras epidemias já haviam sinalizado graves ameaças à saúde coletiva global, como a síndrome respiratória aguda grave (severe acute respiratory syndrom - SARS), em 2002, com 800 mortes; H1N1, em 2009, com 18.500 mortes; síndrome respiratória do oriente médio (Middle East respiratory syndrome MERS), em 2012, com 800 mortes; e Ebola, em 2014, com 11.310 mortes (Anjorin, 2020).

Os meios de comunicação permitem divulgar em tempo real a ameaça que esta pandemia representa, juntamente com os recursos que as culturas têm para enfrentá-la. Se, por um lado, alguns desses meios, como as redes sociais, até por fake news, induzem medo e pânico em grande parte da população, por outro lado, outros deles divulgam informações técnico-científicas relevantes para o enfrentamento da crise, como as plataformas "Novo Coronavírus COVID-19 - Fiocruz", "The Lancet Coronavirus Resource Centre" e "GICA - Gestão da Informação e do Conhecimento na Amazônia". Informações técnico-científicas descrevendo ações preventivas e remediativas necessárias diante da pandemia (cf. Brooks et al., 2020) são fundamentais para orientar o comportamento: dos contaminados, que devem ser mantidos em quarentena; dos não contaminados ou com suspeita de estarem contaminados, que devem ser mantidos em isolamento social; e dos que trabalham em contato direto com os pacientes infectados, que devem usar equipamentos de proteção individual.

Além de informações específicas sobre o patógeno e seus mecanismos de ação fisiológica na progressão da doença, as informações técnico-científicas abordam mais duas questões importantes. A primeira é a adesão da população, em geral, e dos profissionais da saúde, em particular, às estratégias de mitigação dos impactos e da disseminação da doença pandêmica. A segunda é que essa adesão afeta em maior ou menor grau a saúde mental da população, que precisará ser assistida com os tratamentos mais indicados por associações profissionais e científicas de Psicologia, a partir de produções que passaram pelo seu escrutínio crítico. Uma derivação direta dessa segunda questão, relevante ao momento atual, é que há diretrizes e orientações prescrevendo, inclusive, o atendimento psicológico remoto como solução temporária à restrição do contato face a face como parte da medida de isolamento social.

No contexto da relevância social de informações técnico-científicas, o objetivo deste artigo consiste em avaliar aspectos da adesão aos procedimentos de tratamento e controle de pandemias, dos seus impactos sobre a saúde mental e as intervenções psicológicas de escolha. Para atingir esse objetivo, uma revisão narrativa da literatura foi feita, envolvendo buscas bibliográficas no Portal de Periódicos da CAPES de fontes contendo descrições de: impactos de pandemias no comportamento e na saúde mental; adesão da população a estratégias de controle da doença; intervenções psicológicas mais indicadas aos principais agravos à saúde mental em pandemias, incluindo aspectos gerais de sua aplicação remota. Revisões narrativas da literatura constituem sínteses compreensivas de estudos previamente publicados, fornecendo uma perspectiva abrangente, integrada e atualizada sobre determinado tema (Green, Johnson, \& Adams, 2006). Realizar uma revisão narrativa da literatura sobre o impacto de pandemias no comportamento e na saúde mental e sobre intervenções psicológicas de escolha nesse contexto pode contribuir com o trabalho de psicólogos e de outros profissionais que são requeridos a produzir respostas rápidas e efetivas diante dessa situação.

\section{A ADESÃO AOS PROCEDIMENTOS DE TRATAMENTO E CONTROLE DA DOENÇA}

Um aspecto a ser destacado durante pandemias, como a da COVID-19, é a importância da adesão dos governantes, dos profissionais de saúde, dos que foram contaminados e da população em geral aos procedimentos planejados por especialistas para o controle da doença, da sua disseminação e de seus impactos em curto, médio e longo prazos (Zhong et al., 2020). Quando uma vacina não está disponível ainda para a doença (o caso da COVID-19), a tendência da população em se comportar a partir desses procedimentos pode influenciar consideravelmente os resultados imediatos e subsequentes de um surto pandêmico (St. Victor \& Ahmed, 2019).

A possibilidade de as pessoas se comportarem preventivamente implica em modificações sociais que requerem que todos compreendam a situação da pandemia como um problema multifatorial a ser resolvido e cuja solução possa ser atingida (Presti, MecHug, Gloster, Karekla, \& Hayes, 2020). Para isso, estratégias comunitárias de mitigação do contágio são comumente e inicialmente prescritas (Ebrahim, Ahmed, Gozzer, Schlagenhauf, \& Memish, 2020): (a) cancelamento de eventos potencialmente disseminadores da doença; (b) determinação de medidas de distanciamento social para reduzir o contato direto e próximo entre as pessoas em geral; (c) restrição das viagens, aéreas e terrestres, sem comprometer serviços essenciais; (d) quarentena domiciliar voluntária dos membros de famílias afetados não demandantes de internação hospitalar; (e) conhecimento e alterações nos serviços funerários, para minimizar a 
exposição a fluidos corporais contaminados; (f) comunicação direta das autoridades de saúde com a população com informações claras e transparentes, de modo a evitar fake news e pânico.

Quanto à relação autoridade-população, em especial no que diz respeito à comunicação direta de fatos aos cidadãos, é importante atentar ao como o que é pronunciado, enquanto uma instrução, pode controlar comportamentos subsequentes que a autoridade intenciona estarem relacionados à saúde coletiva. Essa importância, também destacada por Beeckman, Hughes, Kissi, Simons e Gourbert (2019) para o caso da dor crônica, está sendo acompanhada em tempo real no mundo (ou deveria estar ocorrendo): os governos focalizam a condição de flexibilidade ou inflexibilidade do seguimento de regras pela população em relação à COVID-19 e decidem o quanto tal condição faz avançar as estratégias coletivas de enfrentamento que eles adotam.

Apreende-se, do que afirmaram Almeida, Ferreira e Moraes (2019), que as instruções devem ser vistas pelo falante como regras (i.e., conjunto de estímulos verbais que especificam contingências) envolvidas na prevenção de doenças, independentemente de a doença já ser uma consequência imediata de comportamentos inseguros à saúde. Esse parece ser o sentido subjacente às urgentes mensagens-chave do artigo de revisão atual sobre impactos psicológicos de quarentenas (Brooks et al., 2020). A principal regra destacada por Brooks et al. para as autoridades em sua pretensão de estabelecer o controle instrucional do macrocomportamento da segurança em saúde numa quarentena é que, em relação à COVID-19, essa comunicação ou informação, além de eficaz, deve ser rápida. A celeridade se justifica porque pessoas precisam entender a situação de calamidade pública que prescreve o isolamento social, a lógica da sua duração (curta em sua necessidade; prorrogada apenas em circunstâncias extremas) e o seu caráter voluntário e altruísta, já que seus impactos psicológicos podem ser piores quando imposta, restringindo a liberdade de ir e vir.

O reforço da comunicação eficaz com a população é a adesão dela às estratégicas de controle da pandemia. Segundo a Organização Mundial de Saúde (World Health Organization, 2003), o controle (medido na diminuição de casos, em especial de contaminados) é a consequência da emissão de comportamentos de adesão, prescritos nas diretrizes dos profissionais da saúde, pautadas na ciência. Nesse aspecto comportamental da adesão estão as tecnologias comportamentais advindas da análise funcional do seguimento de regras na aquisição, manutenção, extinção e supressão de comportamentos relacionados à promoção da saúde e à prevenção de agravos à saúde, a exemplo da adesão ao tratamento do diabetes (e.g., Ferreira \& Fernandes, 2009). Assim, a solução de uma crise sanitária produzida por uma pandemia requer que sejam estabelecidas e mantidas rotinas novas: no trabalho nos setores essenciais, em especial pelos indivíduos que estão no front do trabalho em saúde; na quarentena dos indivíduos infectados, sob os cuidados dos seus familiares; e no isolamento social da população sadia ou não testada para a doença.
A solução de uma crise sanitária pode demorar para ser alcançada, uma vez que as consequências punitivas de não manter essa nova rotina são atrasadas, enquanto o contato social continua, no geral, produzindo seus reforçadores imediatos poderosos. Essa condição é definida por Baum (1994/1999) como "contingência armadilha", conceito que explica a dificuldade em desenvolver e manter comportamentos de autocontrole (cf. Skinner, 1969; Tourinho, 2016) e de auto-observação, que são de extrema importância no contexto atual para evitar maiores proporções da contaminação coletiva. Além do atraso das consequências como fator que dificulta a manutenção de comportamentos relevantes no contexto da COVID-19, o controle restrito de diversas varáveis, como a ausência de vacina e de testes de contágio, resultados inconclusivos dos efeitos de medicamentos no tratamento da doença, o número insuficiente de equipamentos nas unidades hospitalares de terapia intensiva, tornam alguns eventos, como o pico da contaminação, o número de pessoas contaminadas e o número de mortes provocadas pelo vírus, não previsíveis.

Consequências ambientais pouco significativas e independentes do comportamento de um indivíduo (incontroláveis) geram, segundo Maier e Seligman (1976), um padrão menos responsivo e, de acordo com pesquisas experimentais (e.g., Hunziker \& Santos, 2007), dificultam a aprendizagem de comportamentos relevantes. Tal padrão gerado pela incontrolabilidade do ambiente é conhecido por desamparo aprendido, visto como um modelo experimental da depressão. Fassett-Carman, Hankin e Snyder (2019) destacaram a importância da avaliação da controlabilidade e da gravidade dos eventos vivenciados como fatores de risco em potencial para a depressão e outros transtornos psicológicos. Esses aspectos demonstram a relevância de considerar o impacto da pandemia sobre a saúde mental da população.

\section{O IMPACTO DA PANDEMIA SOBRE A SAÚDE} MENTAL

A aproximação de uma doença contagiosa com letalidade alarmante, como a COVID-19, configura uma estimulação aversiva do tipo "dano iminente", descrito por Skinner (1989, p. 7) para definir o tipo de estímulo cuja aversividade é proporcional à sua proximidade e à intensidade do respondente que ele elicia: a ansiedade. Os níveis dela em indivíduos saudáveis e naqueles com transtornos mentais preexistentes podem aumentar e o efeito permanecer em longo prazo (North et al., 2009). Pessoas que continuam trabalhando devem ter cuidado adicional para não transmitir ou contrair o agente patógeno, com destaque para os profissionais da saúde. Pessoas que moram sozinhas também devem se adequar, pois, para elas, o distanciamento social poderá ser ainda mais desafiador. Apesar de North et al. destacarem esses desmembramentos psicossociais das pandemias, segundo a revisão de Brooks et al. (2020) não há consenso sobre quais seriam os preditores de impactos psicológicos negativos de uma quarentena. Por exemplo, o estudo de Taylor, Agho, Stevens e Raphael (2008) assinalou fatores de risco, também presentes na população brasileira, para estresse psicológico na epidemia da gripe equina: idade entre 16-24 anos e baixa escolaridade. Quanto ao impacto em 
profissionais da saúde, os dados não foram consensuais. Já o estudo de Reynolds et al. (2008), realizado durante a quarentena da SARS, destacou sintomas de transtorno do estresse pós-traumático mais graves, mais esquiva e emoções negativas, como culpa, preocupação e desamparo, entre esses profissionais, comparado à população geral (mas ver Hawryluck et al., 2004, que não verificaram esses sintomas entre esses mesmos profissionais).

No que diz respeito ao comportamento do profissional da saúde, deve-se enfatizar as variáveis relacionadas à saúde pública, que absorve um impacto particularmente acentuado dessa pandemia de COVID-19. Nessa absorção, é notório o despreparo dos diversos setores da saúde para lidar com a quantidade de pacientes infectados (cf. Conti, 2020). Os profissionais que estão em contato direto com os problemas causados pela COVID-19, como enfermeiros, médicos, fisioterapeutas, técnicos e agentes da saúde, nunca viveram uma experiência desse tipo. Esse despreparo aumenta a importância de ações emergenciais para reduzir o efeito da crise, que é agravada pela falta de profissionais, de leitos e de diversos materiais, em particular de reagentes para os testes diagnósticos e de aparelhos para a ventilação mecânica na insuficiência respiratória aguda ou crônica agudizada, típica nos casos avançados da COVID-19. As ações emergenciais devem considerar que os profissionais da saúde têm uma vulnerabilidade aumentada para o estresse (e.g., Corrêa, Souza, \& Baptista, 2013), pelas próprias características de suas profissões e dos ambientes onde a exercem.

O estresse é induzido por muitas contingências relacionadas às longas horas de trabalho, ao contato constante com pacientes infectados (e às prováveis mortes deles), ao aumento exacerbado no nível de exigências (e.g., número excessivo de pacientes/horas de trabalho e pressão para ações rápidas e eficazes), à falta de organização e de gestão de recursos, aos baixos níveis de apoio social, às dificuldades para gerir o mal-estar psicológico dos infectados e ao pouco controle da situação (cf. Corrêa et al., 2013; Mello, Reis, \& Ramos, 2018). Como se não bastasse tudo isso, há o medo de contrair a doença e de infectar os membros de sua família. Em suma, os fatores de proteção que amenizam o efeito dessas contingências são escassos.

Alguns profissionais da saúde que entram diretamente em contato com pacientes podem vir a apresentar problemas de saúde mental em curto, médio e longo prazos. Em um estudo desenvolvido por Wu et al. (2009), cerca de $10 \%$ dos participantes (servidores do maior hospital de Beijing) apresentaram estresse pós-traumático em algum período durante os 3 anos posteriores ao surto da SARS2003. Maunder et al. (2006) demonstraram que, de 13 a 26 meses após esse mesmo surto, profissionais da saúde do Hospital Toronto que atenderam pacientes com SARS apresentaram níveis de Burnout, estresse pós-traumático e estresse psicológico significativamente superiores aos dos profissionais dos Hospitais Hamilton, que não trataram pacientes com essa doença. No caso da COVID-19, Lai et al. (2020) demonstraram que, do total de 1.830 profissionais da saúde que participaram do estudo desenvolvido no final de janeiro de 2020, 50,4\% apresentam depressão; 44,6\%, ansiedade; 34\%, insônia; e 71,5\%, estresse. Ainda no contexto da COVID-19, em uma pesquisa realizada com 4.679 médicos e enfermeiros em 348 hospitais chineses, Liu et al. (2020) relataram que $15,9 \%$ dos profissionais apresentavam sofrimento psíquico: $16 \%$ ansiedade e $34,6 \%$ depressão. Dados como esses, podem estar indicando a presença de transtornos mentais, sendo os diagnósticos mais comuns relacionados à COVID-19 (cf. Wang, Zhou, \& Zong, 2020; Zhang et al., 2020), os transtornos da ansiedade, com frequência o transtorno agudo do estresse (TAE) e o transtorno do estresse pós-traumático (TEPT); e os transtornos de humor, frequentemente $\mathrm{O}$ transtorno depressivo maior (TDM).

O TAE se caracteriza por respondentes eliciados (e.g., taquicardia, sudorese) e/ou operantes evocados (e.g. atividades excessivas) pelo contato com eventos aversivos incomuns, como a COVID-19. Sendo essa doença um evento de muita aversividade, um trauma, uma operação motivacional restringe a atenção para a prevenção, controle ou tratamento, o que pode gerar desorientação e dificuldade de discriminação de outros estímulos. Wang et al. (2020) lembraram que isso ocorre em alguns indivíduos mais que outros, e alguns podem nem sequer ter tido contato direto com pacientes infectados ou com ambientes que possam estar contaminados.

O momento do trauma irá diferenciar o TAE do TEPT. Enquanto os sintomas que levam ao diagnóstico do primeiro ocorrem entre 3 a 30 dias da ocorrência do trauma (e.g., trabalho em uma unidade de terapia intensiva lotada de pacientes com a COVID-19), este último é diagnosticado quando esses sintomas permanecem após 30 dias do seu ápice pela exposição ao trauma (Butterbaugh \& Tamas, 2015). Em 2002, a pandemia de SARS produziu sintomas de TEPT semelhantes aos produzidos por outros aversivos extremos, como ataques terroristas e terremotos (Jiang, Nan, Lv, \& Yang, 2020), fato que demonstra a intensidade do trauma produzido pela pandemia. A permanência de sintomas no tempo, segundo Jiang et al., pode estar relacionada à conexão entre o TEPT e a dificuldade em regular emoções, sendo a supressão expressiva e a reavaliação cognitiva as respostas ausentes que mais caracterizam um quadro de desregulação emocional.

No contexto de uma pandemia, o TDM pode evoluir de um lockdown ou de um isolamento social prolongado (Girdhar, Srivastava, \& Sethi, 2020), que pode sinalizar a recessão econômica e com ela, o desemprego. A falta das relações sociais com o isolamento tem a solidão como correlato emocional, especialmente em idosos (Cacioppo, Hawkley, \& Thisted, 2010). Os critérios para diagnóstico do TDM incluem humor deprimido, interesses diminuídos, função cognitiva prejudicada e alterações vegetativas, como no sono, apetite ou sexo. Um fator importante relacionando o TDM e eventos traumáticos, como uma pandemia, conforme apontaram Otte et al. (2016), é que esses eventos afetam os principais sistemas neurobiológicos responsivos ao estresse.

Destaca-se ainda que os impactos psicológicos potenciais da COVID-19 podem ser inferidos de outras pandemias, sendo eles primários, do surto pandêmico da doença em si mesma, ou secundários, por exemplo, a outras perdas, como a perda do emprego e das redes sociais a ele 
envoltas, com a provável recessão econômica que se segue à pandemia. Por isso, Duan e Zhu (2020) lembraram que as intervenções psicológicas na COVID-19 devem ser planejadas a partir de uma avaliação abrangente dos fatores de risco para problemas psicológicos, "incluindo problemas de saúde mental antes de uma crise, luto, autolesões ou lesões aos membros da família, circunstâncias de risco de vida, pânico, separação da família e baixa renda familiar" (p. 301), que podem precipitar ou acompanhar/exacerbar um transtorno mental.

\section{INTERVENÇÕES PSICOLÓGICAS DE ESCOLHA AOS PRINCIPAIS AGRAVOS À SAÚDE MENTAL EM PANDEMIAS}

Os dados dos impactos psicológicos de surtos de doenças pandêmicas demonstram o quanto a intervenção psicológica baseada em evidência é relevante no seu enfrentamento. Nesse contexto, a Ciência do Comportamento é formada por princípios e métodos que favorecem a promoção de ações emergenciais (cf. Leonardi, 2015), por exemplo, as propiciadas pelas terapias de terceira geração (cf. Gouveia, Santos, \& Oliveira, 2015). Da revisão de Books et al. (2020), destacam-se como intervenções psicológicas de escolha em situações de pandemia aquelas direcionadas ao estresse agudo, estresse pós-traumático e desregulação emocional (envolvendo ansiedade, raiva e depressão). A Associação Americana de Psicologia (American Psychological Association - APA) indica os tratamentos psicológicos de escolha para estes transtornos e sintomas, considerados aqui como mais relacionados ao contexto da pandemia da COVID19.

Assim, além da psicoeducação, são destacadas a seguir as intervenções comportamentais embasadas em evidência mais indicadas para $\mathrm{o}$ enfrentamento das consequências da pandemia. Dentre essas indicações, estão as terapias comportamentais de terceira geração: a ativação comportamental, que foca a restauração da variabilidade comportamental perdida nos quadros de depressão; a terapia de aceitação e compromisso, e a terapia comportamental dialética, que permitem focalizar os efeitos rígidos e restritivos induzidos pelo medo e ansiedade, desenvolver habilidades de mindfulness e regular emoções (Dornelles \& Sayago, 2015; Presti et al., 2020). Tais habilidades não só permitem intervir em estados emocionais após a crise, mas também antes e durante o período da sua ocorrência.

Os serviços de saúde que fornecem apoio psicológico aos profissionais da saúde devem incluir o treinamento de estratégias adequadas para o manejo da ansiedade e do estresse. Quando o suporte organizacional é ofertado, ele é percebido de forma positiva, e gera um sentimento de valorização e satisfação, uma percepção de que a organização/empresa, ou todo o sistema, está comprometida e preocupada com o bem-estar dos seus agentes (Mello et al., 2018). Isso contribui para a motivação e o comprometimento com o trabalho, melhorando o desempenho profissional. $\mathrm{O}$ apoio psicológico com o fornecimento de estratégias de enfrentamento do estresse favorece o desenvolvimento da autoconfiança, a sensação da capacidade de desempenho em atividades específicas. Conforme apontaram Corrêa et al. (2013), quando um profissional tem suporte afetivo e social, aumenta a probabilidade de uma relação satisfatória dele com o trabalho, que passa a ser visto como um contexto que considera a satisfação das suas necessidades.

Intervenções psicoeducativas objetivam fornecer suporte e apoio ao cuidado de pacientes e/ou cuidadores por meio de informações e/ou instruções sobre determinada doença/condição de saúde, bem como sobre estratégias para manejar situações decorrentes dela. Tais intervenções contribuem para a prevenção e conscientização em saúde e podem ser direcionadas tanto ao paciente quanto aos familiares ou cuidadores (Colom \& Vieta, 2004). Em um estudo de revisão de literatura que objetivou avaliar o uso da psicoeducação em casos de doenças somáticas e mentais (e.g. hipertensão, câncer, depressão), Lemes e Ondere Neto (2017) identificaram que essa intervenção tem mostrado efeitos positivos. Apesar de ser uma intervenção que tem, em geral, como público-alvo, pacientes ou seus familiares, os autores destacam que profissionais da saúde também podem se beneficiar dela, em especial quando enfrentando doenças novas, como a COVID-19. Na literatura sobre intervenções psicológicas para o manejo de situações decorrentes da pandemia de COVID-19, a psicoeducação tem sido sugerida como parte importante de um pacote de intervenções (e.g Brooks et al., 2020; Sá-Serafim, do Bú, \& Lima-Nunes, 2019), porque pode mitigar os efeitos negativos da pandemia sobre a saúde mental de profissionais da saúde, pacientes e familiares (Lemes \& Ondere Neto, 2017), facilitando a identificação e manejo de sintomas (cf. Brooks et al., 2020), como o estresse emocional, a ansiedade e a estigmatização social decorrente do diagnóstico da doença.

A Tabela 1 contém as informações do site da APA (https://www.div12.org/treatments/) sobre as demais intervenções psicológicas indicadas para os impactos psicológicos da COVID-19 e referências com detalhamento da sua técnica. A intensidade da base de evidência dos tratamentos - suportada por estudos bem delineados e conduzidos por pesquisadores independentes - é "forte" em todos os tipos listados, exceto na terapia de aceitação e compromisso, em que é "modesta" para o transtorno misto de ansiedade e depressão, entretanto, "forte" para dor crônica.

A pandemia da COVID-19, além de permitir o destaque de um conjunto de intervenções psicológicas, evidenciou o potencial do atendimento psicológico remoto, ao mesmo tempo em que o Congresso Nacional no Brasil regulamentou a telemedicina. No que diz respeito aos atendimentos feitos no Brasil, eles devem estar de acordo com a Resolução CFP $n^{\circ} 11 / 2018$, que dispõe sobre a prestação de serviços psicológicos realizados por meio de tecnologias da informação e da comunicação (TICs). De acordo essa resolução, que autoriza a prestação de serviços psicológicos realizados por meios de TICs de forma síncrona e assíncrona, o profissional deve efetuar um cadastro prévio no Conselho Federal de Psicologia, especificar os recursos tecnológicos utilizados para garantir um cadastro prévio no Conselho Federal de Psicologia, especificar os recursos tecnológicos utilizados para garantir o sigilo das informações do cliente e ter sempre em vista as disposições do Código de Ética Profissional do Psicólogo. 
Tabela 1. Intervenções psicológicas indicadas para os impactos psicológicos da COVID-19, sintoma diagnóstico de indicação, premissa e duração do tratamento. A Coluna 1 inclui citações de manuais de referência técnica.

\begin{tabular}{|c|c|c|c|c|}
\hline Tratamento & $\begin{array}{c}\text { Sintoma } \\
\text { Diagnóstico } \\
\text { de indicação }\end{array}$ & Premissa & Essência & Duração \\
\hline $\begin{array}{l}\text { Exposição Prolongada } \\
\text { (PE) } \\
\text { (Foa, Hembree, \& } \\
\text { Rothbaum, 2007) }\end{array}$ & $\begin{array}{l}\text { Trauma } \\
\text { Estresse pós- } \\
\text { traumático }\end{array}$ & $\begin{array}{l}\text { A exposição repetida a } \\
\text { estímulos do tipo } \\
\text { pensamentos, sentimentos e } \\
\text { situações relacionados ao } \\
\text { trauma da pandemia pode } \\
\text { ajudar a reduzir o limiar } \\
\text { desses estímulos em eliciar } \\
\text { angústia }\end{array}$ & $\begin{array}{l}\text { PE consiste em } \\
\text { exposições, com } \\
\text { bloqueio de esquiva, a } \\
\text { estímulos imaginais } \\
\text { para contatar a memória } \\
\text { traumática relacionada } \\
\text { à doença e processar } \\
\text { essa experiência de } \\
\text { contato, bem como, a } \\
\text { estímulos in vivo } \\
\text { seguros relacionados ao } \\
\text { trauma }\end{array}$ & 8-15 sessões \\
\hline $\begin{array}{l}\text { Ativação } \\
\text { Comportamental } \\
\text { (BehavioralActivation, } \\
\text { BA) } \\
\text { (Lejuez, Hopko, 2013) }\end{array}$ & Depressão & $\begin{array}{l}\text { Em isolamento social, as } \\
\text { pessoas saem da rotina, se } \\
\text { isolam e deprimem; com o } \\
\text { tempo, essa esquiva agrava } \\
\text { o humor deprimido, pois } \\
\text { elas perdem oportunidades } \\
\text { de reforço positivo em } \\
\text { atividades e em } \\
\text { experiências de prazer ou } \\
\text { de domínio possíveis nas } \\
\text { limitações impostas pela } \\
\text { pandemia }\end{array}$ & $\begin{array}{l}\text { BA aumenta o contato } \\
\text { do paciente com fontes } \\
\text { de reforço positivo, } \\
\text { possíveis durante a } \\
\text { pandemia, ajudando-o a } \\
\text { ser mais ativo; ao fazê- } \\
\text { lo, melhora o seu } \\
\text { contexto de vida, } \\
\text { mesmo em isolamento; } \\
\text { concentra-se na } \\
\text { clarificação dos valores } \\
\text { que orientam a seleção } \\
\text { de atividades; e na } \\
\text { identificação de } \\
\text { processos que inibem a } \\
\text { ativação } \\
\text { comportamental e/ou } \\
\text { promovem respostas de } \\
\text { solução de problemas }\end{array}$ & $\begin{array}{l}\text { Versão longa: } \\
\text { 20-24 sessões; } \\
\text { Versão breve: } \\
\text { 8-15 sessões }\end{array}$ \\
\hline $\begin{array}{l}\text { Resolução de } \\
\text { Problemas } \\
\text { (RP) } \\
\text { (Nezu, Nezu, D'Zurilla, } \\
2006) \text {. }\end{array}$ & $\begin{array}{l}\text { Depressão } \\
\text { Outros }\end{array}$ & $\begin{array}{l}\text { A história das pessoas no } \\
\text { lidar de modo eficaz ou não } \\
\text { com eventos estressantes } \\
\text { em geral pode afetar o grau } \\
\text { em que experimentam ou } \\
\text { podem vir a experimentar } \\
\text { angústia relacionada à } \\
\text { COVID-19 }\end{array}$ & $\begin{array}{l}\text { RP é uma intervenção } \\
\text { tipo "guarda-chuva" } \\
\text { que aumenta o ajuste } \\
\text { adaptativo aos } \\
\text { problemas e ao estresse } \\
\text { da vida na pandemia } \\
\text { pelo treino de respostas } \\
\text { motoras, afetivas e } \\
\text { cognitivas para a } \\
\text { solução eficaz de } \\
\text { problemas que surgem } \\
\text { em função da doença }\end{array}$ & $\begin{array}{l}\text { Aprox.12 } \\
\text { sessões; mas } \\
\text { mudanças } \\
\text { efetivas foram } \\
\text { observadas em } \\
\text { menos de } 4 \\
\text { sessões ou mais } \\
\text { de } 12 \text {, a } \\
\text { depender do } \\
\text { tipo de estilo de } \\
\text { solução de } \\
\text { problemas e do } \\
\text { grau de } \\
\text { desregulação } \\
\text { emocional do } \\
\text { paciente }\end{array}$ \\
\hline $\begin{array}{l}\text { Terapia de Aceitação e } \\
\text { Compromisso } \\
\text { (Acceptance and } \\
\text { Commitment Therapy, } \\
\text { ACT) } \\
\text { (Eifert \& Forsyth, } \\
2005)\end{array}$ & $\begin{array}{l}\text { Ansiedade e } \\
\text { depressão }\end{array}$ & $\begin{array}{l}\text { ACT é sobre aceitação e } \\
\text { mudança em relação à } \\
\text { doença/isolamento social, } \\
\text { um incentivo ao abandono } \\
\text { da luta para mudar } \\
\text { pensamentos e emoções } \\
\text { indesejadas e, em vez disso, } \\
\text { ao concentrar-se no }\end{array}$ & $\begin{array}{l}\text { ACT visa alterar a } \\
\text { relação do paciente com } \\
\text { pensamentos, } \\
\text { sentimentos, memórias } \\
\text { e sensações físicas } \\
\text { indesejadas ou temidas, } \\
\text { relativas à pandemia. } \\
\text { Estratégias de aceitação }\end{array}$ & $\begin{array}{l}\text { Aprox. } 12 \\
\text { sessões }\end{array}$ \\
\hline
\end{tabular}


momento presente e atuar em direção aos valores

\author{
e mindfulness são \\ usadas para ensiná-lo a \\ reduzir a esquiva \\ experiencial e a fusão a \\ esses estímulos \\ privados e aumentar o \\ foco no presente e na \\ flexibilidade \\ psicológica. Ele \\ aprende a clarificar \\ metas e valores e a se \\ comprometer com a \\ mudança \\ comportamental

$\begin{array}{ll}\begin{array}{l}\text { DBT ensina ao paciente } \\ \text { habilidades }\end{array} & \begin{array}{l}\text { Varia, mas } \\ \text { geralmente dura } \\ \text { comportamentais }\end{array} \\ \text { de 1 a 1,5 anos, } \\ \text { diversificadas, } & \text { incluindo } \\ \text { adaptadas ao novo } & \text { sessões } \\ \text { contexto da COVID-19, } & \text { semanais } \\ \text { como mindfulness, } & \text { individuais e } \\ \text { tolerância à angústia, } & \text { coletivas } \\ \text { eficácia interpessoal e } & \text { (grupos de } \\ \text { regulação emocional } & \text { treinamento de } \\ & \text { habilidades) }\end{array}$ \\ Varia, mas \\ de 1 a 1,5 anos, \\ incluindo \\ essões \\ individuais e \\ coletivas \\ treinamento de \\ habilidades)
}

$\begin{array}{ll}\text { Terapia } & \begin{array}{l}\text { Desregulação } \\ \text { emocional, } \\ \text { Comportamental }\end{array} \\ \text { Dialética } & \begin{array}{l}\text { presente em } \\ \text { muitos }\end{array} \\ \text { Therapy - DBT) } & \begin{array}{l}\text { transtornos, } \\ \text { como no }\end{array} \\ \text { (Linehan, 2014) } & \text { TEPT }\end{array}$

Algumas pessoas

experimentam emoções

muito intensas e

desreguladas em relação à

pandemia e, para não sofrer

assim, elas podem tender a

ser extremas e impulsivas

(e.g., autolesão, uso de

drogas ou suicídio). Falhas

na consciência/aceitação de

emoções interferem no

desenvolvimento de

estratégias mais eficazes de

enfrentamento do

sofrimento intenso relativo

à pandemia
Diferentemente da resolução anterior $\left(\mathrm{CFP} \mathrm{n}^{\circ}\right.$ $11 / 2012$ ), a resolução em vigor determina que as sessões não fiquem limitadas ao caráter experimental, como também não dita restrições ao número de sessões realizadas. Dessa maneira, nos últimos 2 anos, a modalidade on-line tem ganho espaço na atuação do psicólogo no Brasil, apresentando vantagens, como disponibilidade, acessibilidade, conveniência, privacidade e redução de estigma (Cartreine, Ahern, \& Locke, 2010). A terapia online demonstra ser tão eficaz quanto a presencial em termos de resultados alcançados (cf. Miclea, Miclea, Ciuca, \& Budau, 2010), pois, conforme demonstraram Preschl, Maercker e Wagner (2011), a relação terapêutica não apresentou diferenças significativas ao se comparar as duas formas de prover a terapia.

No atual cenário de riscos da COVID-19, o CFP (2020a) recomendou, no dia 24 de março, que os atendimentos na modalidade presencial fossem suspensos até o término do isolamento social; posteriormente, atendimentos presenciais indispensáveis foram autorizados com as devidas regras de distanciamento social e demais cuidados, como manter o consultório higienizado e ventilado. Nesse contexto, o atendimento on-line é mais seguro, permitindo que as pessoas em isolamento social ou em quarentena tenham acesso ao serviço psicológico. Nesse caso, tal serviço também se torna uma opção para os profissionais da linha de frente no enfrentamento da pandemia, visto que, como citado anteriormente, além de serem expostos ao risco de contraírem a doença e de contaminarem seus familiares e colegas, são acometidos por sofrimento psicológico. O CFP (2020b) também suspendeu os Art. $3^{\circ}$, Art. $4^{\circ}$, Art. $6^{\circ}$, Art. $7^{\circ}$ e Art. $8^{\circ}$ da Resolução de 11 de maio de 2018 em nova Resolução no dia 26 de março de 2020, o que possibilita que pessoas e grupos em situação de urgência e emergência sejam atendidos pelos meios permitidos pelas TICs.

\section{CONSIDERAÇÕES FINAIS}

A situação de pandemia causada pela COVID-19 alterou o contexto mundial afetando a política e a economia de diversas nações e a vida dos indivíduos e das famílias, no que diz respeito às condições de saúde. Em situações desse tipo, o foco das intervenções necessita abranger não apenas a saúde física e o combate ao agente patogênico, mas também a saúde mental da população. Aspectos tais como prevenção da contaminação pelo vírus, tratamento dos que foram contaminados e suas famílias, e o suporte psicológico aos profissionais da saúde não podem ser negligenciados. Além disso, a incontrolabilidade e a gravidade dos eventos, confirmadas pelo alto índice de pessoas contaminadas e de falecimentos, são aspectos de extrema relevância e que necessitam ser levados em consideração pelos que se valem dos meios de comunicação para promover mudança de comportamento da população em relação à pandemia.

Os transtornos mentais mais prevalentes nessas circunstâncias e que requerem atenção dos profissionais da 
Psicologia são transtornos da ansiedade, com frequência o transtorno agudo do estresse, o transtorno do estresse póstraumático, e os transtornos de humor, frequentemente o transtorno depressivo maior. Intervenções psicoeducativas e o apoio psicológico on-line são alternativas de intervenção passíveis de serem executadas por psicólogos, no enfrentamento do estresse e no favorecimento do desenvolvimento da autoconfiança e do autocontrole. Além disso, são relevantes intervenções psicológicas tanto para amenizar o sofrimento mental quanto para prevenir agravos, decorrentes da situação de pandemia.

Diante da vulnerabilidade de algumas das pessoas envolvidas na pandemia, a Associação Americana de Psicologia destacou modelos terapêuticos que, até então no Brasil, eram realizados em atendimento presencial, podem ser realizados de forma remota. Uma vez que a terapia online apresenta vantagens e proporciona a aplicação de intervenções e desenvolvimento da relação terapêutica, os diferentes modelos de terapias comportamentais podem orientar a prática de psicólogos no atendimento remoto, não descartando a necessidade de estudos que investiguem sua efetividade e adaptabilidade à cultura brasileira. Embora a situação mundial seja calamitosa, a pandemia constitui um desafio que acaba por contribuir para o aperfeiçoamento da prática do psicólogo, bem como de outras áreas de estudo/intervenção, com destaque para a saúde pública e a medicina.

\section{DECLARAÇÃO DE CONFLITO DE INTERESSES}

Os autores declaram que não há conflito de interesses relativos à publicação do artigo.

\section{CONTRIBUIÇÃO DE CADA AUTOR}

Certificamos que todos os autores participaram suficientemente do trabalho para tornar pública sua responsabilidade pelo conteúdo. A contribuição de cada autor pode ser atribuída como se segue: E. Borloti, V. B. Haydu, N. Kienen e M. R. J. Zacarin foram responsáveis pela proposta e pela redação do artigo.

\section{DIREITOS AUTORAIS}

Este é um artigo aberto e pode ser reproduzido livremente, distribuído, transmitido ou modificado, por qualquer pessoa desde que usado sem fins comerciais. $\mathrm{O}$ trabalho é disponibilizado sob a licença Creative Commons 4.0 BY-NC.

\section{$(c)$ EY-NC}

\section{REFERÊNCIAS}

Almeida, F. P., Ferreira, E. A. P., \& Moraes, A. J. P. de (2019). Effects of instruction and self-monitoring on adherence to treatment of juvenile systemic lupus erythematosus. Temas em Psicologia, 27, 173-187. doi: 10.9788/TP2019.1-13

Anjorin, A. A. (2020). The coronavirus disease 2019 (COVID-19) pandemic: A review and an update on cases in Africa. Asian Pacific Journal of Tropical Medicine, 13, 1-5. doi: 10.4103/1995-7645.281612
Baum, W. M. (1999). Compreender o behaviorismo: comportamento, cultura e evolução (M. T. A. Silva, M. A. Matos, G. Y. Tomanari \& E. Z. Tourinho Trads.). Porto Alegre: Artmed. (Versão original publicada em 1994).

Beeckman, M., Hughes, S., Kissi, A., Simons, L. E., \& Goubert, L. (2019). How an understanding of our ability to adhere to verbal rules can increase insight into (mal)adaptive functioning in chronic pain. The Journal of Pain, 20, 1141-1154. doi 10.1016/j.jpain.2019.02.013

Brooks, S. K., Webster, R. K., Smith, L. E., Woodland, L., Wessely, S., Greenberg, N., \& Rubin, G. J. (2020). The psychological impact of quarantine and how to reduce it: Rapid review of the evidence. The Lancet, 395(10227), 912-920. doi: 10.1016/S01406736(20)30460-8

Butterbaugh, S., \& Tamas, R. L. (2015). Posttraumatic stress disorder vs acute stress disorder. In Duong, T., Tamas, R. L. \&, Ureste, P. (Orgs.), Psychiatry morning report: Beyond the pearls. E-Book. USA: Elsevier.

Cacioppo, J. T., Hawkley, L. C., Thisted, R. A. (2010). Perceived social isolation makes me sad: 5-year crosslagged analyses of loneliness and depressive symptomatology in the Chicago Health, Aging, and Social Relations Study. Psychology and Aging, 25(2), 453- 453-63. doi: 10.1037/a0017216

Cartreine, J. A., Ahern, D. K., \& Locke, S. E. (2010). A roadmap to computer-based psychotherapy in the United States. Harvard Review of Psychiatry, 18(2), 8095. doi: 10.3109/10673221003707702

Colom, F., \& Vieta, E. (2004). Melhorando o desfecho do transtorno bipolar usando estratégias não farmacológicas: O papel da psicoeducação. Revista Brasileira de Psiquiatria, 26, 47-50. doi 10.1590/s1516-44462004000700011

Conselho Federal de Psicologia (2012). Resolução CFP N 011/2012. Recuperado de: http://site.cfp.org.br/wpcontent/uploads/2012/07/Resoluxo_CFP_nx_01112.pdf

Conselho Federal de Psicologia (2018). Resolução No ${ }^{o} 11$, DE 11 de maio de 2018. Recuperado de: https://site.cfp.org.br/wpcontent/uploads/2018/05/RESOLU\%C3\%87\%C3\%83 O-N\%C2\%BA-11-DE-11-DE-MAIO-DE-2018.pdf

Conselho Federal de Psicologia (2020a). Ofício-Circular $n^{\circ}$ 40/2020/GTec/CG-CFP. https://site.cfp.org.br/wpcontent/uploads/2020/03/SEI_CFP-0214041-

Of\%C3\%ADcio-Circular_.pdf

Conselho Federal de Psicologia (2020b). Resolução CFP N ${ }^{\circ}$ 4, de 26 de março de 2020. Recuperado de: http://www.in.gov.br/en/web/dou/-/resolucao-n-4-de26-de-marco-de-2020-250189333

Conti, T. V. (2020). Crise Tripla do Covid-19: um olhar econômico sobre políticas públicas de combate à pandemia. Recuperado de: shorturl.at/jyFZ9

Corrêa, R. Z. A., Souza, M. S., \& Baptista, M. N. (2013). Vulnerabilidade ao estresse no trabalho e qualidade de vida de enfermeiros. Psicologia Argumento, 31, (75). doi: 10.7213/psicol.argum.31.075.DS02 
Dornelles, V. G., \& Sayago, C. W. (2015). Terapia comportamental dialética: Princípios e bases do tratamento. In P. Lucena-Santos, J. Pinto-Gouveia, \& M. da S. Oliveira (Orgs.), Terapias Comportamentais de Terceira Geração: Guia para profissionais (p. 440473). Novo Hamburgo, RS: Sinopsys.

Duan, L., \& Zhu, G. (2020). Psychological interventions for people affected by the COVID-19 epidemic. The Lancet Psychiatry, 7(4), 300-302. doi: 10.1016/S22150366(20)30073-0

Ebrahim, S. H., Ahmed, Q. A., Gozzer, E., Schlagenhauf, P., \& Memish, Z. A. (2020). Covid-19 and community mitigation strategies in a pandemic. $B M J, 368, \mathrm{~m} 1066$. doi: 10.1136/bmj.m1066

Eifert, G. H., \& Forsyth J. P. (2005). Acceptance and commitment therapy for anxiety disorders: A practitioner's treatment guide to using mindfulness, acceptance, and value-based behavior change strategies. Oakland, CA: New Harbinger.

Fassett-Carman, A., Hankin, B. L., \& Snyder, H. R. (2019). Appraisals of dependent stressor controllability and severity are associated with depression and anxiety symptoms in youth. Anxiety, Stress, \& Coping, 32(1), 32-49. doi: 10.1080/10615806.2018.1532504

Ferreira, E. A. P., \& Fernandes, A. L. (2009). Treino em auto-observação e adesão à dieta em adulto com diabetes Tipo 2. Psicologia: Teoria e Pesquisa, 25, 629. 636. doi: 10.1590/S0102-37722009000400019

Foa, E. B., Hembree, E., \& Rothbaum B. O. (2007) Prolonged exposure therapy for PTSD: Emotional processing of traumatic experiences - Therapist guide (1st Edition), New York: Oxford.

Girdhar, R., Srivastava, V., \& Sethi, S. (2020). Managing mental health issues among elderly during COVID-19 pandemic. Journal of Geriatric Care and Research, 7(1), 29-32. Recuperado de: https://www.academia.edu/42715576/Managing_menta 1_health_issues_among_elderly_during_COVID_ 19_pandemic

Gouveia, J. P., Santos, L. P., \& Oliveira, M. S. (2015). Terapias comportamentais de terceira geração: Guia para profissionais. Novo Hamburgo: Sinopsys.

Green, B. N., Johnson, C. D., \& Adams, A. (2006). Writing narrative literature reviews for peer-reviewed journals: Secrets of the trade. Journal of Chiropractic Medicine, 5(3), 101-117. doi: 10.1016/S0899-3467(07)60142-6

Hawryluck, L., Gold, W. L., Robinson, S., Pogorski, S., Galea, S., \& Styra, R. (2004). SARS control and psychological effects of quarantine, Toronto, Canada. Emerging Infectious Diseases, 10(7), 1206-1212. doi: 10.3201/eid1007.030703

Hunziker, M. H. L., \& Santos, C. V. (2007). Learned helplessness: Effects of response requirement and interval between treatment and testing. Behavioural Processes, 76(3), 183-191. doi: 10.1016/j.beproc.2007.02.012

Jiang, H-J., Nan, J., Lv, Z-Y., \& Yang, J. (2020). Psychological impacts of the COVID-19 epidemic on Chinese people: Exposure, post-traumatic stress symptom, and emotion regulation. Asian Pacific
Journal of Tropical Medicine. Recuperado de: http://www.apjtm.org/preprintarticle.asp?id=281614

Lai, J., Ma, S., Wang, Y., Cai, Z., Hu, J., Wei, N., ... Hu, S. (2020). Factors associated with mental health outcomes among health care workers exposed to coronavirus disease 2019. JAMA Network Open, 3(3): e203976. doi: 10.1001/jamanetworkopen.2020.3976

Lejuez, C. W., \& Hopko, D. R. (2013). Behavioral Activation (BA) Program: Treatment Manual. Recuperado de: https://www.div12.org/treatment/behavioral-activationfor-depression/

Lemes, C. B., \& Ondere Neto, J. (2017). Aplicações da psicoeducação no contexto da saúde. Temas em Psicologia, 25(1), 17-28. doi: 10.9788/TP2017.1-02

Leonardi, J. L. (2015). O lugar da terapia analíticocomportamental no cenário internacional das terapias comportamentais: Um panorama histórico. Perspectivas em Análise do Comportamento, 6(2), 119-131. doi: 10.18761/pac.2015.027

Levin, P. J., Gebbie E. N., \& Qureshi, K. (2007). Can the health-care system meet the challenge of pandemic flu? Planning, ethical, and workforce considerations. Public Health Reports, 122, 573-578. Recuperado de: www.ncbi.nlm.nih.gov/pmc/articles/PMC1936949/pdf/ phr122000573.pdf

Linehan, M. M. (2014). DBT Skills Training Handouts and Worksheets. New York, NY: Guilford.

Liu, Z., Han, B., Jiang, R., Huang, Y., Ma, C., Wen, J., ... Ma, Y. (2020). Mental Health Status of Doctors and Nurses During COVID-19 Epidemic in China. SSRN Electronic Journal. doi: 10.2139/ssrn.3551329

Maier, S. F., \& Seligman, M. E. P. (1976). Learned helplessness: Theory and evidence. Journal of Experimental Psychology: General, 105(1), 3-46. doi: 10.1037/0096-3445.105.1.3

Maunder, R. G., Lancee, W. J., Balderson, K. E., Bennett, J. P., Borgundvaag, B., Evans, S., Fernandes, C. M., ... Wasylenki, D. A. (2006). Long-term psychological and occupational effects of providing hospital healthcare during SARS outbreak. Emerging Infectious Diseases, 12(12), 1924-1932. doi: 10.3201/eid1212.060584

Mello, R.de C. C., Reis, L. B., \& Ramos, F. P. (2018) Estresse em profissionais de enfermagem: Importância da variável clima organizacional. Revista Interinstitucional de Psicologia, 11(2), 193-207. doi: 10.36298/gerais2019110202

Miclea, M., Miclea, S., Ciuca, A. M., \& Budau, O. (2010). Computer-mediated psychotherapy. Present and prospects. A developer perspective. Cognition, Brain, Behavior: An Interdisciplinary Journal, 14(3), 185-208. Recuperado http://www.cbbjournal.ro/index.php/ro/2010/65-143/439-computer-mediated-psychotherapy-present-andprospects-a-developer-perspective

Nezu, A. M., Nezu, C. M., \& D'Zurilla, T. (2006). Solving life's problems. A 5-step guide to enhanced well-being. New York: Springer.

North, C. S., Pfefferbaum, B., Vythilingam, M., Martin, G J., Schorr, J. K., Boudreaux, A. S., ... Hong, B. A. 
(2009). Exposure to bioterrorism and mental health response among staff on Capitol Hill. Biosecurity and Bioterrorism, 7(4), 379-388. doi: 10.1089/bsp.2009.0031

Otte, C., Gold, S. M., Penninx, B. W., Pariante, C. M., Etkin, A., Fava, M., ... Schatzberg, A. F. (2016). Major depressive disorder. Nature Reviews Disease Primers, 2, 16065. doi: 10.1038/nrdp.2016.65

Preschl, B., Maercker, A., \& Wagner, B. (2011). The working alliance in a randomized controlled trial comparing online with face-to-face cognitivebehavioral therapy for depression. BMC Psychiatry, 11(189), 2-10. doi: 10.1186/1471-244X-11-189

Presti, G., McHugh, L., Gloster, A., Karekla, M., \& Hayes, S. C. (2020). The dynamics of fear at the time of COVID-19: A contextual behavioral science perspective. Clinical Neuropsychiatry, 17(2), 65-71. doi: $10.36131 / \mathrm{CN} 20200206$

Reynolds, D. L., Garay, J. R., Deamond, S. L., Moran, M. K., Gold, W., \& Styra, R. (2008). Understanding, compliance and psychological impact of the SARS quarantine experience. Epidemiology and Infection, 136(7), 997-1007. doi: 10.1017/S0950268807009156

Sá-Serafim, R., do Bú E., Lima-Nunes A. (2020). Manual de diretrizes para atenção psicológica nos hospitais em tempos de combate ao COVID-19. Revista Saúde \& Ciência, $8(2), \quad 1-24$ https://doi.org/10.35572/rsc.v8i2.876

Skinner, B. F. (1969). Contingencies of reinforcement: A theoretical analysis. New York: Appleton-CenturyCrofts.

Skinner, B. F. (1989). Recent issues in the analysis of behavior. Columbus, $\mathrm{OH}$ : Merrill.

St. Victor, G., \& Ahmed, S. (2019). The importance of culture in managing mental health response to pandemics. In Huremovié, D. (Org.), Psychiatry of pandemics: A mental health response to infection outbreak (pp. 55-56). New York: Springer.

Taylor, M. R., Agho, K. E., Stevens, G. J., \& Raphael, B. (2008). Factors influencing psychological distress during a disease epidemic: Data from Australia's first outbreak of equine influenza. BMC Public Health, 8, 113. doi: $10.1186 / 1471-2458-8-347$

Tourinho, E. Z. (2016) Mundo interno e autocontrole. Revista Brasileira de Análise do Comportamento, 2(1), 21-36. doi: 10.18542/rebac.v2i1.800

Wang, C., Zhou, J., \& Zong, C. (2020). Two cases report of epidemic stress disorder to novel coronavirus pneumonia. Asian Journal of Psychiatry, 51, 102070. doi: 10.1016/j.ajp.2020.102070

World Health Organization (2003). Adherence to long-term therapies: Evidence for action. Geneva. Recuperado de: http://www.who.int/chp/knowledge/publications/adher ence_full_report.pdf

Wu, P., Fang, Y., Guan, Z., Fan, B., Kong, J., Yao, Z., ... Hoven, C. W. (2009). The psychological impact of the SARS epidemic on hospital employees in China: Exposure, risk perception, and altruistic acceptance of risk. Revue Canadienne de Psychiatrie, 54(5), 302-311. doi: 10.1177/070674370905400504
Zhang, F., Shang, Z., Ma, H., Jia, Y., Sun, L., Guo, X., .. Liu, W. (2020). High risk of infection caused posttraumatic stress symptoms in individuals with poor sleep quality: A study on influence of coronavirus disease (COVID-19) in China. MedRxiv. doi: 10.1101/2020.03.22.20034504

Zhong, B.-L., Luo, W., Li, H.-M., Zhang, Q.-Q., Liu, X.G., Li, W.-T., \& Li, Y. (2020). Knowledge, attitudes, and practices towards COVID-19 among Chinese residents during the rapid rise period of the COVID-19 outbreak: A quick online cross-sectional survey. International Journal of Biological Sciences, 16(10), 1745-1752. doi: 10.7150/ijbs.45221

Submetido em: 03/05/2020 Aceito em: 08/06/2020 